\title{
Thiodiethylene glycol based polyesters: synthesis and thermal characterization
}

\author{
N. Lotti ${ }^{1}$, L. Finelli $^{1}$, M. Messori ${ }^{2}$, A. Munari $^{1}$ \\ ${ }^{1}$ Dipartimento di Chimica Applicata e Scienza dei Materiali, Università di Bologna, \\ Viale Risorgimento 2, 40136 Bologna, Italy; Fax ++390512093220; \\ nadia.lotti@mail.ing.unibo.it \\ 2Dipartimento di Ingegneria dei Materiali e dell'Ambiente, Università di Modena e \\ Reggio Emilia, Via Vignolese, 905/A - 41100 Modena, Italy; Fax ++390592056243; \\ messori.massimo@unimore.it
}

(Received: 24 November, 2005; published: 17 May 2006)

\begin{abstract}
Poly(thiodiethylene terephthalate) (PSDET), poly(thiodiethylene adipate) (PSDEA), poly(diethylene terephthalate) (PDET) and poly(diethylene adipate) (PDEA) for comparison were synthesized and characterized in terms of chemical structure and molecular weight. The thermal behavior was examined by thermo gravimetric analysis and differential scanning calorimetry. All the polymers showed a good thermal stability, even though lower for the sulfur-containing polyesters. At room temperature they appeared as semicrystalline materials, except PDEA, which was an oil; the effect of substitution of ether oxygen atoms with sulfur ones was a lowering in the $T_{g}$ value, an increment of melting temperature and an increase of crystallization rate. The results were explained as due to the presence of flexible $\mathrm{C}-\mathrm{S}-\mathrm{C}$ bonds in the polymeric chain.
\end{abstract}

\section{Introduction}

In recent years the study of the synthesis and the properties of sulfur-containing polyesters have received a renewed impulse, due to some specific applications proposed for these materials. The polyesters, mainly based on thiodipropionic, thiodiisobutyric, dithiodibutyric, dithiodibenzoic or dithiodiacetic acid, have been claimed to be plasticizers for poly(vinyl chloride) or rubber modifiers; they can also be used with the addition of elemental sulfur (i.e. by heating a mixture of sulfur and thiopolyesters caulking compounds or plastics) to obtain highways marking stripes. Moreover, it is well known that the presence of sulfur atoms in a polymeric chain can improve some important properties, such as refractive index, biodegradability, etc. As a consequence, several copolyesters containing sulfur atoms in the main chain or in side chains were prepared and studied [1-8]. In addition, polyesters based on thiodipropionic acid were also synthesized and the solid or semi-solid electrolytes obtained by dissolving appropriate lithium salts in them were studied [9-11]. These electrolytes showed rather good characteristics for use in rechargeable lithium batteries.

In this regard, herein, poly(thiodiethylene adipate) (PSDEA), poly(thiodiethylene terephthalate) (PSDET), and poly(diethylene adipate) (PDEA) and poly(diethylene terephthalate) (PDET) for comparison, were synthesized in our laboratories and characterized in terms of molecular and thermal properties, in order to analyze the effect of the substitution of the ether oxygen atoms with sulfur ones. 


\section{Results and discussion}

At room temperature all the polyesters synthesized are semicrystalline solids, except PDEA, which appears as oil. The solubility of the polymers was checked in various solvents: all the samples showed a good solubility at room temperature in the most common organic solvents, i.e. chloroform, tetrachloroethane, methylene chloride, etc. Before molecular and thermal characterizations, PSDEA, PDET and PSDET were carefully purified by the following procedure: after dissolution in chloroform and precipitation in methanol, they were recovered by filtration and again dissolved and reprecipitated twice. The polymers synthesized are listed in Tab. 1, which also presents the number-average molecular weight $\left(M_{n}\right)$ data obtained by GPC technique.

Tab. 1. Molecular and thermal characterization data.

\begin{tabular}{|c|c|c|cc|cccccc|}
\hline \multicolumn{2}{|c|}{} & \multicolumn{10}{c|}{$1^{\text {st }}$ scan } & \multicolumn{10}{c|}{$2^{\text {nd }}$ scan } \\
\hline Polymer & $M_{n}$ & $\begin{array}{c}T_{i d} \\
\left({ }^{\circ} \mathrm{C}\right)\end{array}$ & $\begin{array}{c}T_{m} \\
\left({ }^{\circ} \mathrm{C}\right)\end{array}$ & $\begin{array}{c}\Delta H_{m} \\
(\mathrm{~J} / \mathrm{g})\end{array}$ & $\begin{array}{c}T_{g} \\
\left({ }^{\circ} \mathrm{C}\right)\end{array}$ & $\begin{array}{c}\Delta C_{p} \\
\left(\mathrm{~J} / \mathrm{g}^{\circ} \mathrm{C}\right)\end{array}$ & $\begin{array}{c}T_{c} \\
\left({ }^{\circ} \mathrm{C}\right)\end{array}$ & $\begin{array}{c}\Delta H_{c} \\
(\mathrm{~J} / \mathrm{g})\end{array}$ & $\begin{array}{c}T_{m} \\
\left({ }^{\circ} \mathrm{C}\right)\end{array}$ & $\begin{array}{c}\Delta H_{m} \\
(\mathrm{~J} / \mathrm{g})\end{array}$ \\
\hline PDEA & 18900 & 335 & - & - & -43 & 0.682 & - & - & - & - \\
PSDEA & 22400 & 358 & 63 & 80 & -60 & 0.530 & -14 & 50 & 60 & 51 \\
PDET & 27400 & 390 & 78 & 43 & 26 & 0.368 & - & - & - & - \\
PSDET & 21400 & 332 & 112 & 41 & 14 & 0.384 & 98 & 17 & 109 & 18 \\
\hline
\end{tabular}

The chemical structure of all polyesters was determined by FT-IR and ${ }^{1} \mathrm{H}-\mathrm{NMR}$ spectroscopy. As far as FT-IR analysis is concerned, the characteristic carbonyl stretching frequencies of the ester groups appeared at 1710 and $1100 \mathrm{~cm}^{-1}(\mathrm{C}=\mathrm{O}$ and $\mathrm{C}-\mathrm{O}$ ), respectively, whereas the $\mathrm{C}-\mathrm{H}$ bending vibration of the $\mathrm{CH}_{2}-\mathrm{S}$ group was located at $1450 \mathrm{~cm}^{-1}$; moreover, it was evident the intensity reduction of the band centered at $3400 \mathrm{~cm}^{-1}$, corresponds to the stretching vibration of hydroxyl groups of diethylene glycol and thiodiethylene glycol, that reacted or have been removed from the reaction system during the syntheses. The disappearance of most of the $\mathrm{OH}$ groups can be therefore considered as an evidence of the fact that the polymerization reaction took place. In order to have an understanding into the chemical structure, the ${ }^{1} \mathrm{H}-\mathrm{NMR}$ investigation on the samples was performed. In all cases, the spectra were found to be consistent with the expected structure, the chemical shift assignments $(\delta, \mathrm{ppm})$ being the following: PDEA: $\delta 4.21\left(\mathrm{t}, 4 \mathrm{H}^{\mathrm{c}}\right), \delta 3.67$ $\left(\mathrm{t}, 4 \mathrm{H}^{\mathrm{d}}\right), \delta 2.34\left(\mathrm{t}, 4 \mathrm{H}^{\mathrm{b}}\right), \delta 1.65\left(\mathrm{~m}, 4 \mathrm{H}^{\mathrm{a}}\right)$; PSDEA: $\delta 4.20\left(\mathrm{t}, 4 \mathrm{H}^{\mathrm{c}}\right), \delta 2.77\left(\mathrm{t}, 4 \mathrm{H}^{\mathrm{d}}\right), \delta 2.34$ $\left(\mathrm{t}, 4 \mathrm{H}^{\mathrm{b}}\right), \delta 1.64\left(\mathrm{~m}, 4 \mathrm{H}^{\mathrm{a}}\right) ;$ PDET: $\delta 8.00\left(\mathrm{~s}, 4 \mathrm{H}^{\mathrm{c}}\right), \delta 4.51\left(\mathrm{t}, 4 \mathrm{H}^{\mathrm{b}}\right), \delta 3.87\left(\mathrm{t}, 4 \mathrm{H}^{\mathrm{a}}\right)$; PSDET: $\delta 8.08\left(\mathrm{~s}, 4 \mathrm{H}^{\mathrm{c}}\right), \delta 4.52\left(\mathrm{t}, 4 \mathrm{H}^{\mathrm{b}}\right), \delta 2.98\left(\mathrm{t}, 4 \mathrm{H}^{\mathrm{a}}\right)$ (see the chemical structures reported in the Experimental Section).

The polyesters were afterwards examined by thermogravimetric analysis and differential scanning calorimetry. The investigation on the thermal stability was carried out both in air and under nitrogen atmosphere. Fig. 1 shows the thermogravimetric curves of the samples in air. 


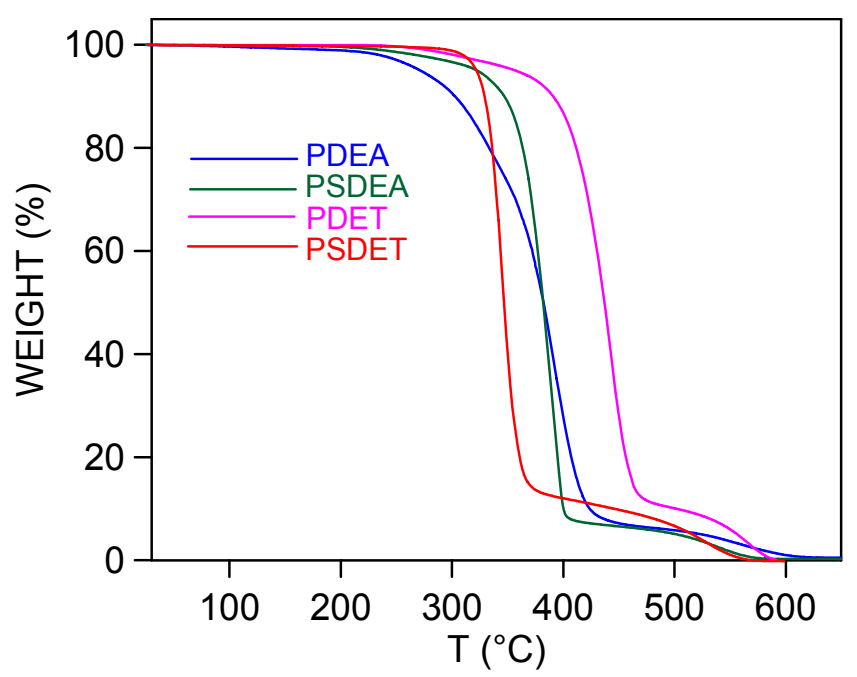

Fig. 1. Thermogravimetric curves in air (10 deg/min).

It can be seen that in all cases the weight loss takes place practically in one-step and is $100 \%$. Moreover, as evidenced by the temperature of initial decomposition $\left(T_{\text {id }}\right)$ reported in Tab. 1, all the samples are characterized by a relatively high thermal stability, the $T_{\text {id }}$ values ranging from 330 to $390^{\circ} \mathrm{C}$. As far as the effect of the substitution of the ether oxygen atoms with sulfur ones on the thermal stability of these materials is concerned, a distinction between aliphatic and aromatic polyesters has to be made: in fact, in the case of aromatic polyesters the presence of sulfur atoms along the polymeric chain causes a decrement of the thermal stability which can be ascribed to the lower energy of the S-C bond as compared to O-C one. As to the aliphatic polyesters, PDEA and PSDEA are characterized by a comparable thermal stability, except in the low temperature range where the ether oxygencontaining polyester appears to be less stable. This behavior could be ascribed to volatilization process of low molecular weight compounds present in the polymer. Lastly, from the comparison between the TGA curves of PDET and PDEA, one can see that the aromatic polyester is more thermally stable than the aliphatic one, as expected on the basis of the general decomposition mechanism proposed for such polymers [12]. In fact, the formation of vinyl and carboxyl groups by ester scission and by a cyclic elimination process occurs more easily in flexible aliphatic polyesters [12]. The corresponding sulfur-containing polymers show on the contrary an unusual behavior: PSDEA indeed turns out to be characterized by a higher thermal stability than PSDET. This trend could be explained on the basis of a different thermal degradation mechanism. In order to investigate these results more deeply, further experiments are currently being carried out. Similar trends were obtained by means of TGA measurements carried out under nitrogen atmosphere.

With regard to calorimetric results, the samples being characterized by high and similar $M_{n} \mathrm{~s}$, an influence of molecular weight on the glass transition and melting phenomena of the polymers synthesized can be excluded. It is well established that the melting behavior of a polymer is affected by its previous thermal history and therefore, in order to provide the same heat treatment to all the samples investigated, prior to thermal analysis the specimens have been aged for a very long time (about 6 months) at room temperature in desiccators. The DSC traces of such samples are reported in Figure 2 and the data obtained in Tab. 1. 

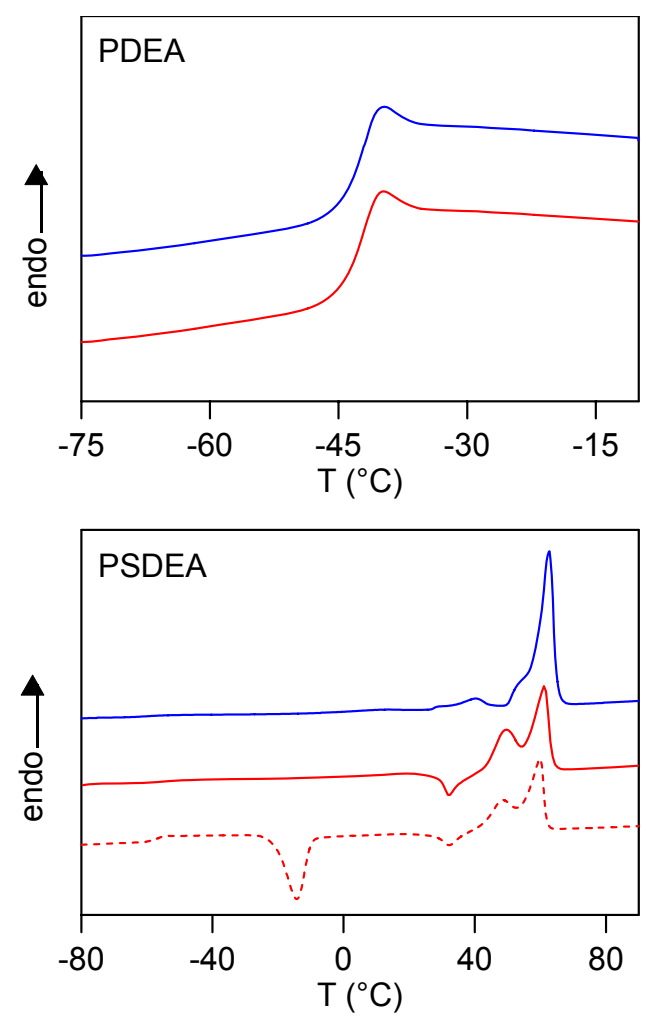
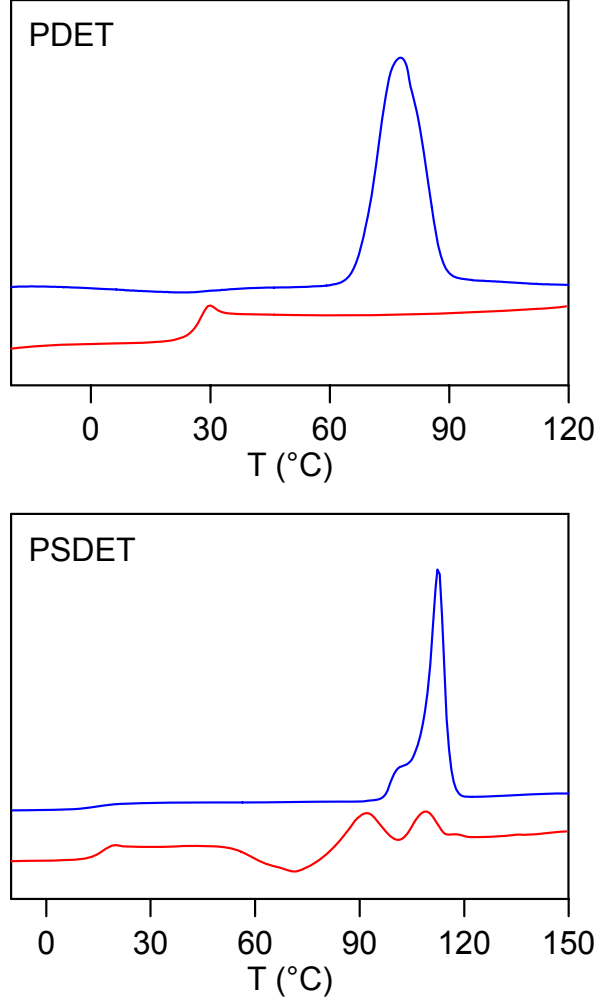

Fig. 2. (-) $1^{\text {st }}$ scan; (-) $2^{\text {nd }}$ scan after melt quenching inside DSC; (--) $2^{\text {nd }}$ after melt quenching outside calorimeter in liquid nitrogen.

Concerning PDEA, the DSC curve is characterized only by an intense endothermal baseline deviation associated with the glass transition phenomenon. On the contrary, in all other cases, a melting endotherm is also evident. In order to analyze the effect of substitution of the ether oxygen atoms with sulfur ones on the melting point, the $T_{m}$ values of the sulfur-containing polyesters can be compared to those of the ether oxygen-containing polymers. As can be seen from the Fig. 2 and from the data collected in Tab. 1, PSDET melts at higher temperature than PDET, although the former polymer is characterized by a lower polarity than the latter one. As already reported by other authors [13], this anomalous behavior may be explained on the basis of a higher packing efficiency in PSDET with respect to PDET. As a matter of fact, the packing efficiency is higher when the values of the Van der Waals radii of the atoms or groups forming the crystallizable polymer chain are similar. In PSDET, the sulfur atoms and the neighbor $\mathrm{CH}_{2}$ groups are indeed characterized by similar Van der Waals radius, which are 1.8 and $2.0 \AA$, respectively; whereas in PDET the oxygen atom has a Van der Waals radius which is lower $(1.4 \AA)$. Moreover, the aliphatic PSDEA polyester melts at a considerable lower temperature with respect to the aromatic PSDET, as expected on the basis of the reduction in the number of conformations available to the polymeric chain, resulting from the replacement of an aliphatic segment with a ring structure.

As is well known, a partially crystalline material usually exhibits a different glass transition behavior than the completely amorphous analogs. In fact, although some conflicting results are reported in the literature [14], crystallinity usually acts like cross linking and raises $T_{g}$ through its restrictive effect on the segmental motion of amorphous polymer chains. Therefore, in order to study the influence of chemical structure on the glass transition of a polymer, the phenomenon should be examined in the total absence of crystallinity. With this view, all the samples under investigation 
were subjected to rapid cooling (quenching) from the melt (see the Experimental section for the details). The DSC curves after melt quenching are shown in Fig. 2: both the DSC traces of the ether oxygen-containing samples are characterized only by an intense endothermal baseline deviation associated with the glass transition, the presence of crystallization and melting processes being not evident. As far as the sulfur-containing polymers are concerned, a distinction between the aliphatic and the aromatic one has to be made: in fact, the calorimetric curve of PSDEA is characterized by an endothermal baseline deviation associated to the glass transition phenomenon followed by melting enodothermic peaks at higher temperature, indicating that the sample cannot be frozen into a completely amorphous state by quenching inside DSC and therefore is partially crystalline. In order to completely prevent crystallization and obtain PSDEA in a completely amorphous condition, the polymer was quenched outside the calorimeter cell by immersion in liquid nitrogen as quickly as possible. As is well known, such method permits a faster cooling with respect to that obtained inside the DSC equipment. The corresponding DSC trace is reported in Fig. 2 which is characterized by a glass transition phenomenon and processes of crystallization and melting of comparable heats. It can be therefore concluded that in this case a completely amorphous sample is obtained. The DSC trace of PSDET after melt quenching inside DSC shows a glass transition followed by an exothermal "cold crystallization" peak and a melting endotherms at higher temperature. As the enthalpy of crystallization very well compares with the corresponding heat of fusion, the polymer can be considered completely amorphous.

As it can be seen from the data collected in Tab. 1, the $T_{g}$ values are significantly affected by the chemical characteristics of the polymers: the aromatic polyesters present a higher $T_{g}$ value than the corresponding aliphatic ones, as expected on the basis of the higher rigidity of the structure. In fact, as it is well known, the incorporation along a polymeric chain of aromatic rings causes a considerably increment in the chain stiffness. The stiff ring indeed restricts the rotation in the backbone, consequently reducing the number of conformations that the polymer chain can adopt. By analyzing the experimental $T_{g}$ values, it also results that the substitution of the ether oxygen atoms by sulfur causes a significant decrement of $T_{g}$. This trend can be again explained on the basis of the higher flexibility of the polymer chain due to the presence of sulfur atoms which, being characterized by larger dimensions with respect to the oxygen ones, form S-C bonds longer than the O-C ones. This behavior has been already observed for other sulfur-containing polymers $[15,16]$. It is noteworthy that the second scans of both the sulfur-containing poly esters are characterized by double melting peaks. In order to investigate the nature of the phenomenon, the effect of the scanning rate on the melting behavior of these samples has been analyzed.

In Fig. 3, the DSC curves of PSDEA scanned at different heating rates after isothermal crystallization at $-20^{\circ} \mathrm{C}$ are reported: two endotherms are observed in all cases: for sake of clarity, the peak at lower temperature has been marked I, whereas that at higher temperature II. It can be observed that (i) the endotherm II moves to higher temperatures as the heating rate decreases and (ii) the ratio of the area of the first melting peak to the second one increases as the heating rate is increased, suggesting that the multiple melting is due to a mechanism based on melting and recrystallization of less perfect crystallites into thicker crystals, followed by a final melting process at higher temperature. 


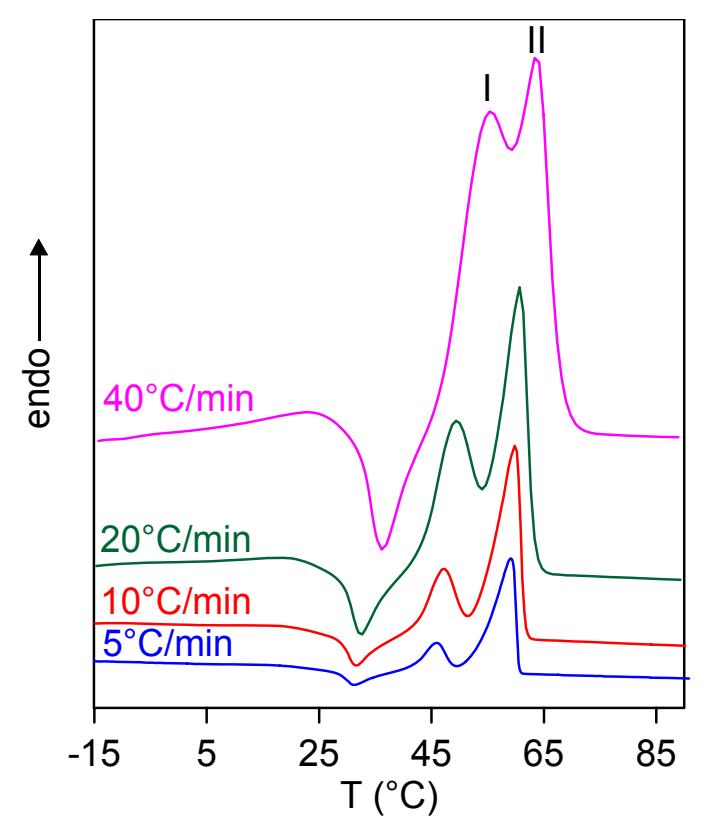

Fig. 3. DSC melting endotherms of PSDEA scanned at the indicated heating rate after isothermal crystallization at $-20^{\circ} \mathrm{C}$. The curves have not been corrected for changes in the thermal lag with the heating rate.

In order to clarify the nature of the double melting peak, wide-angle X-ray measurements on samples isothermally crystallized from the melt at different temperatures were carried out and the results reported elsewhere [17]. WAXS data indicated that the unit cell parameters remained unchanged, regardless of the crystallization temperature. As a consequence, the double melting peak was ascribed to melting and recrystallization processes occurring during the calorimetric run [17]. As far as PSDET polyester is concerned, as shown in Fig. 4, the melting behavior appears to be strongly affected by heating rate: two crystallization and two melting phenomena are observed at the lowest heating rates $\left(5\right.$ and $\left.10^{\circ} \mathrm{C} / \mathrm{min}\right)$; at the intermediate heating rates $\left(20\right.$ and $\left.30^{\circ} \mathrm{C} / \mathrm{min}\right)$, the crystallization peak at higher temperature (labeled in the Fig. as $T_{c 2}$ ) is not evident anymore; lastly, at the highest heating rate $\left(40^{\circ} \mathrm{C} / \mathrm{min}\right)$ only a baseline deviation associated with the glass transition phenomenon appears, indicating that the macromolecules don't have enough time to rearrange and crystallize during the heating scan.

The DSC behavior of PSDET is therefore quite peculiar: the two crystallization and two melting phenomena observed at the lowest heating rates can suggest the existence of two different crystalline populations with different stability. Moreover, taking into account that the enthalpy associated with crystallization $\left(\Delta H_{c}\right)$ is comparable to that required for melting $\left(\Delta H_{m}\right)$ (the total values resulting from the two crystallization and the two melting processes are considered), PSDET is totally amorphous after melt quenching, the crystalline phase only developing above $T_{g}$ in "cold crystallization" processes. Hence the crystal phases that melt must be developed during the heating run: the low melting temperature phase in the first exothermal process, the high melting temperature crystals during the second crystallization phenomenon. 


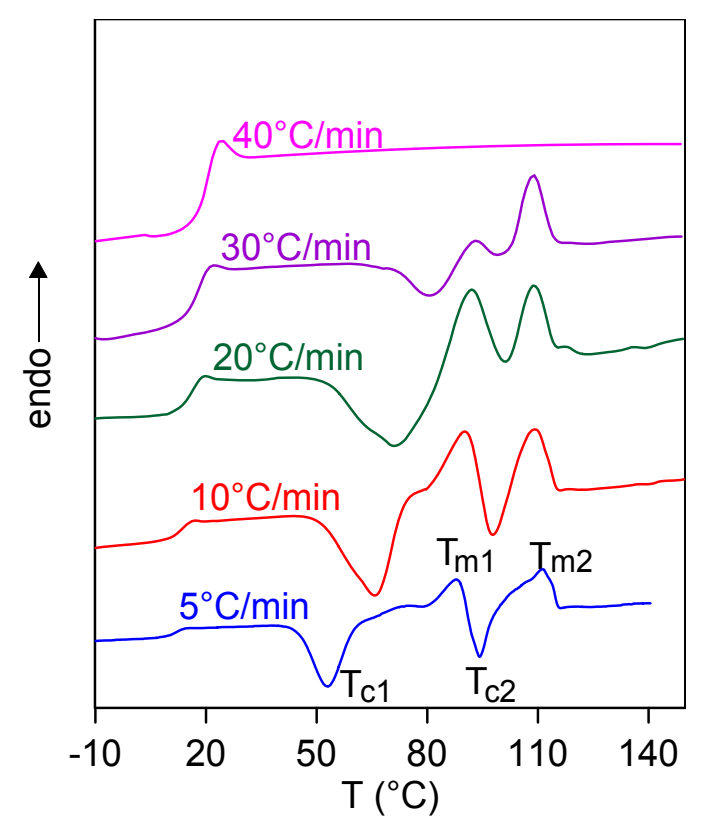

Fig. 4. Calorimetric curves after melt quenching inside DSC of PSDET scanned at the indicated heating rates.

Nevertheless, the shape and the location of the double endotherms observed at the different heating rates can also suggest that the low-melting crystalline phase $\left(T_{m 1}\right)$ has a tendency to melt and reorganize into better crystals, the high melting peak $\left(T_{m 2}\right)$ corresponding in all curves to the fusion of the best crystals re-arranged during the heating scan. Moreover, as usually found, the reorganization process happens if the heating rate is lower. Due to the complexity of the phenomenon, an accurate investigation by using differential scanning calorimetry, X-ray diffraction and polarizing optical microscopy has been carried and the results have been reported elsewhere $[18,19]$. The complex multiple melting behavior of PSDET was correlated to the existence of two different crystal structures, each of them however subjected to reorganization effects during the heating run. The XRD measurements permitted to point out that the formation of each form is selective and depends strongly on the crystallization conditions, i.e. from the glassy or molten state [18, 19].

The second scans obtained after melt quenching in DSC of all the samples under investigation also shows that the chemical structure of the macromolecules has a strong influence on the ability to crystallize. In the case of sulfur-containing polyesters, it can be evicted that the aliphatic sample crystallizes faster than the aromatic one during the cooling step, as expected on the basis of a higher chain flexibility which favors the chain folding. A comparison between the sulfur-containing polymer and the corresponding oxygen-containing one (PDEA in fact does not crystallize), displays that the former crystallize faster than the latter. This behavior can be explained as due to the presence of sulfur atoms along the polymeric chain which make the macromolecules more flexible and mobile and consequently the rearrangements of the macromolecules into 3-dimensional order can occur more easily.

Lastly, as far as PDET polyester is concerned, it is worth noting that no crystallization phenomenon has been observed during the cooling step from the melt, even if slow cooling rates are used. This behavior has been already evidenced in a previous paper [20, 21], where the influence of various solvents, polymer concentration and crystallization temperature on the crystallization process of the polyester has been 
described. In particular [20], the precipitation from solution (method used by us to purify the polymer), i.e. a "solvent crystallization", has been described as an efficacious procedure that allows crystallization of a larger fraction of the sample. As a matter of fact, PDET reveals to be a semicrystalline material after purification, as evidenced by the $1^{\text {st }}$ scan of Fig. 2 .

\section{Conclusions}

The data obtained on the aromatic and aliphatic polyesters synthesized display that small modifications of the chemical structure, such as the substitution of ether oxygen atoms with sulfur ones, can lead to significant variations in the thermal properties. In particular:

- the thermal stability decreases, due to the lower energy value of the S-C bonds, as compared to O-C ones;

- the glass transition temperature decreases, due to the higher flexibility of the sulfur-containing macromolecular chains;

- the melting point increases, due to the higher packing efficiency;

- the ability to crystallize increases, due to higher chain flexibility which favors the chain folding.

The above-mentioned effects can all be ascribed to the larger dimensions of sulfur atoms with respect to oxygen ones.

In conclusion, the results reported here confirm that the chemical structure is a very important factor in controlling the final properties of a polymeric material, which has to be taken into account in order to obtain products useful for specific applications.

\section{Experimental part}

\section{Reagents}

Dimethylterephthalate (DMT), dimethyladipate (DMA), diethylene glycol (DEG), thiodiethylene glycol (TDEG) and titanium tetrabutoxide $\left(\mathrm{Ti}(\mathrm{OBu})_{4}\right)$ (Aldrich) were reagent grade products; DMT, DMA and TDEG were used as supplied, whereas DEG and $\mathrm{Ti}(\mathrm{OBu})_{4}$ were distilled before use.

\section{Synthesis of polymer samples}

Poly(thiodiethylene terephthalate) (PSDET), poly(diethylene terephthalate) (PDET), poly(thiodiethylene adipate) (PSDEA) and poly(diethylene adipate) (PDEA) polymers were synthesized in bulk starting from DMT or DMA and a glycol (DEG or TDEG using a $10 \% \mathrm{~mol} \%$ excess of the glycol with respect to DMT or DMA), according to the kind of polyester to be prepared, employing $\mathrm{Ti}(\mathrm{OBu})_{4}$ as catalyst (about $0.2 \mathrm{~g}$ of $\mathrm{Ti}(\mathrm{OBu})_{4} / \mathrm{kg}$ of polymer). The syntheses were carried out in a $200 \mathrm{~mL}$ stirred glass reactor, with a thermostatted silicon oil bath; temperature and torque were continuously recorded during the polymerization. The polymers were obtained according to the usual two-stage polymerization procedure. In the first stage, under pure nitrogen flow, the temperature was fixed at 180 or $200^{\circ} \mathrm{C}$ and maintained there until more than $90 \%$ of the theoretical amount of methanol was distilled off (typically, about 3 hour). In the second stage the pressure was reduced, in order to facilitate the removal of glycol in excess and the temperature was kept at 200 or $220^{\circ} \mathrm{C}$, until a torque constant value was measured. Lower reaction temperatures were employed 
for the synthesis of aliphatic polyesters, in order to minimize the thermal degradation reactions. The polymers obtained, because of the use of $\mathrm{Ti}(\mathrm{OBu})_{4}$ as catalyst and the high temperature, which favor redistribution reactions, are statistical in molecular weight distribution. The monomeric units of the synthesized polyesters are the following:

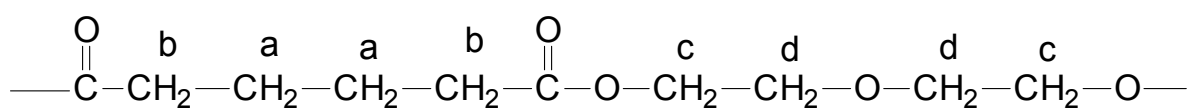
DEA

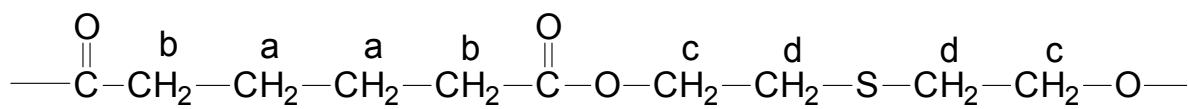
SDEA<smiles>COCCOCCOC(=O)c1ccc(C(C)=O)cc1</smiles>
DET<smiles>COCC[CH]CSCCOC(=O)c1ccc(C(C)=O)cc1</smiles>
SDET

\section{Infrared and ${ }^{1} \mathrm{H}-\mathrm{NMR}$ spectroscopy}

Infrared spectra were recorded on an IFS48 Bruker Fourier Transform Infrared (FTIR) Spectrophotometer, using film samples cast on a sodium chloride plate from chloroform solutions.

The chain structures of the polymers were determined by means of ${ }^{1} \mathrm{H}-\mathrm{NMR}$ spectroscopy. Polymer samples were dissolved $(15 \mathrm{mg} / \mathrm{mL})$ in chloroform-d with $0.03 \%(\mathrm{v} / \mathrm{v})$ tetramethylsilane added as an internal standard. The measurements were carried out at room temperature, employing a Varian XL-300 instrument.

\section{Gel-permeation chromatography}

Molecular weight data were obtained by gel-permeation chromatography at $30^{\circ} \mathrm{C}$ using a 1100 Hewlett Packard system with a refractive index (at $254 \mathrm{~nm}$ wavelength) as detector, equipped with PL gel $5 \mu$ Mixed C column (300/7.5 length/i.d., in mm). Chloroform was used as eluent with a $0.75 \mathrm{~mL} / \mathrm{min}$ flow, and sample concentrations of about $10 \mathrm{mg} / \mathrm{mL}$ were applied. A molecular weight calibration curve was obtained with several monodisperse polystyrene standards in the range of molecular weight $3000-100000$.

\section{Thermal analysis}

Calorimetric measurements were carried out by means of a Perkin Elmer DSC7 instrument equipped with a liquid sub ambient accessory and calibrated with high purity standards (indium and cyclohexane). Unless otherwise indicated, weighed samples (c.a. $10 \mathrm{mg}$ ) were encapsulated in aluminum pans and heated to about $40^{\circ} \mathrm{C}$ above fusion temperature at a rate of $20^{\circ} \mathrm{C} / \mathrm{min}$ (first scan) and then rapidly 
quenched at $-100^{\circ} \mathrm{C}$. Finally, they were reheated from $-100^{\circ} \mathrm{C}$ to a temperature well above the fusion temperature of the sample at a heating rate of $20^{\circ} \mathrm{C} / \mathrm{min}$ (second scan). The glass-transition temperature $T_{g}$ was taken as the midpoint of the heat capacity increment $\Delta c_{p}$ associated with the glass-to-rubber transition. The melting temperature $\left(T_{m}\right)$ was determined as the peak value of the endothermal phenomenon in the DSC curve; when multiple endotherms were observed, the highest peak temperature was taken as $T_{m}$. The enthalpy of fusion $\left(\Delta H_{m}\right)$ was determined from the total area of DSC endotherms. When a crystallization phenomenon was observed during the heating scan, the crystallization temperature $\left(T_{c}\right)$ was taken as the temperature of the main exothermic peak and the enthalpy of crystallization $\left(\Delta H_{c}\right)$ was determined from the total area of DSC exotherm. Repeated measurements on each sample showed excellent reproducibility.

Thermogravimetric analysis was carried out both in air and under nitrogen atmosphere using a Perkin Elmer TGA7 apparatus (gas flow: $50 \mathrm{~mL} / \mathrm{min}$ ) at $10^{\circ} \mathrm{C} / \mathrm{min}$ heating rate up to $900{ }^{\circ} \mathrm{C}$.

\section{References}

[1] Kultys, A.; Podkoscielny, W.; J. Appl. Polym. Sci. 1990, 39, 715.

[2] Kultys, A.; Podkoscielny, W.; J. Appl. Polym. Sci. 1993, 49, 1615.

[3] Kultys, A.; Podkoscielny, W.; J. Appl. Polym. Sci. 1996, 61,1781.

[4] Kultys, A.; J. Polym. Sci., Part A: Polym. Chem. 1997, 35, 547.

[5] Kultys, A.; J. Polym. Sci., Part A: Polym. Chem. 1997, 35, 2231.

[6] Kultys, A.; Podkoscielny, W.; Pikus, S.; J. Polym. Sci., Part A: Polym. Chem. 1998, 36, 2359.

[7] Lotti, N.; Finelli, L.; Milizia, T.; Munari, A.; Manaresi, P.; Eur. Polym. J. 2000, 36, 929.

[8] Lotti, N.; Finelli, L.; Siracusa, V.; Munari, A.; Gazzano, M.; Polymer 2002, 43, 4355.

[9] Manaresi, P.; Bignozzi, MC.; Pilati, F.; Munari, A.; Mastragostino, M.; Meneghello, L.; Chiolle, A.; Polymer 1993, 34, 2422.

[10] Mastragostino, M.; Meneghello, L.; Manaresi, P.; Bignozzi, MC.; Pilati, F.; Munari, A.; Polymer 1994, 35, 845.

[11] Bandiera, M.; Manaresi P.; Munari, A.; Borghini, MC.; Mastragostino, M.; Eur. Polym. J. 1997, 33, 1679.

[12] Goodman, I.; In Enciclopedia of Polymer Science and Engeneering; Wiley: New York, 1988, p. 1, $2^{\text {nd }}$ ed., vol. 12.

[13] Riande, E.; Guzman, J.; San Roman, J.; J. Chem. Phys. 1980, 72(9), 5263.

[14] Boyer, RF.; Rub. Chem. Tech. 1963, 36, 1303.

[15] Berti, C.; Celli, A.; Marianucci, E.; Eur. Polym. J. 2002, 38, 1281.

[16] Berti, C.; Celli, A.; Marianucci, E.; Tannini, M.; Eur. Polym. J. 2005, 41, 1812.

[17] Finelli, L.; Lotti, N.; Munari, A.; Gazzano, M.; Malta, V.; J. Polym. Sci: Part B: Polym. Phys. 2004, 42, 553.

[18] Fichera, AM.; Finelli, L.; Gazzano, M.; Lotti, N.; Munari, A.; Macromol. Chem. Phys. 2004, 205, 63.

[19] Fichera, AM.; Finelli, L.; Gazzano, M.; Lotti, N.; Malta, V.; Munari, A.; Macromol. Chem. Phys. 2004, 205, 1752.

[20] Guzman, J.; Fatou, JG.; Eur. Polym. J. 1978, 14, 943.

[21] Piana, U.; Zizzoli, M.; Buchanan, CM.; Polymer, 1995, 36, 373. 